\title{
Transport de sédiments fins dans le bassin de Marennes-Oléron : mise en place d'un modèle mathématique
}

\author{
O. Raillard ( $\left.{ }^{1}\right)$, P. Le Hir et P. Lazure
}

IFREMER, Centre de Brest, BP 70, 29280 Plouzané, France

\section{Introduction.}

Le secteur littoral de Marennes-Oléron est une zone d'intérêt économique de première importance : c'est en effet le premier bassin ostréicole de France, dont l'activité est profondément influencée par les remises en suspension et les évolutions bathymétriques (HERAL et al., 1984). Du strict point de vue hydrosédimentaire, le secteur présente aussi beaucoup d'intérêt, puisqu'il est le siège de mouvements simultanés de bancs de sable et de vasières, générés par un forçage combiné houle/courant lié à la marée et aux événements météorologiques. A priori, on peut considérer que les remises en suspension sont principalement provoquées par les houles du large et les clapots induits par des vents locaux, tandis que les courants de marée, modulés par les mêmes vents, sont responsables des transports de matériaux dans le bassin.

On réalise ainsi la complexité des études hydrosédimentaires à mener pour comprendre les transports de sédiments, et en particulier la difficulté d'intégrer les multiples processus concernés, que seul un modèle mathématique permet de coupler de manière déterministe.

Un tel outil peut en outre s'intégrer dans une modélisation plus globale de l'écosystème où peuvent être associés les processus physiques et biologiques tels que production primaire, croissance des mollusques etc. (BACHER, 1989, RAILLARD, 1991).

Compte tenu de la prédominance des sédiments fins dans les matières en suspension (MES) et de leur rôle privilégié comme vecteur de nutriment ou de contaminant, ce sont les sédiments cohésifs qui sont considérés dans un premier temps. L'objet du présent article est de présenter la mise en place d'un modèle de transport de sédiments vaseux dans le bassin de Marennes-Oléron, de décrire les schémas d'évolution sous l'action de la marée et de tester le rôle possible des houles et clapots dans les bilans sédimentaires à moyen terme, avec un souci de validation des résultats par des observations in situ, lorsqu'elles sont disponibles.

\section{Présentation de la baie de Marennes-Oléron}

Le secteur étudié comprend le pertuis d'Antioche, largement ouvert aux influences océaniques, et, au sud de l'estuaire de la Charente, le bassin de Marennes-Oléron, limité au sud par le pertuis de Maumusson (fig. l). La

(') Adresse actuelle: CETIIS, 24 Bd Paul Vaillant-Couturier, 94200 Ivry-sur-Seine, France.

\section{Fine sediment transport in the Bay of Marennes-Oléron} Development of a mathematical model

The bay of Marennes-Oléron (Atlantic coast, France) is submitted to tides, swells and wind-induced waves. An hydrodynamical and sedimentological model has been developed to compute the effects of these forcings on cohesive sediments.

Model and measurements are in good agreement to simulate the turbidity fluctuations during the fortnightly tidal cycle. Adding the effect of wind waves improves the turbidity results within the tidal cycle: this is due to the large tidal flats where small depths allow for sediment resuspension under frequent wind waves action. The sediment budgets are qualitatively in good agreement with observations, but the tide effect seems overestimated. The wave action is likely to partially balance this effect. 


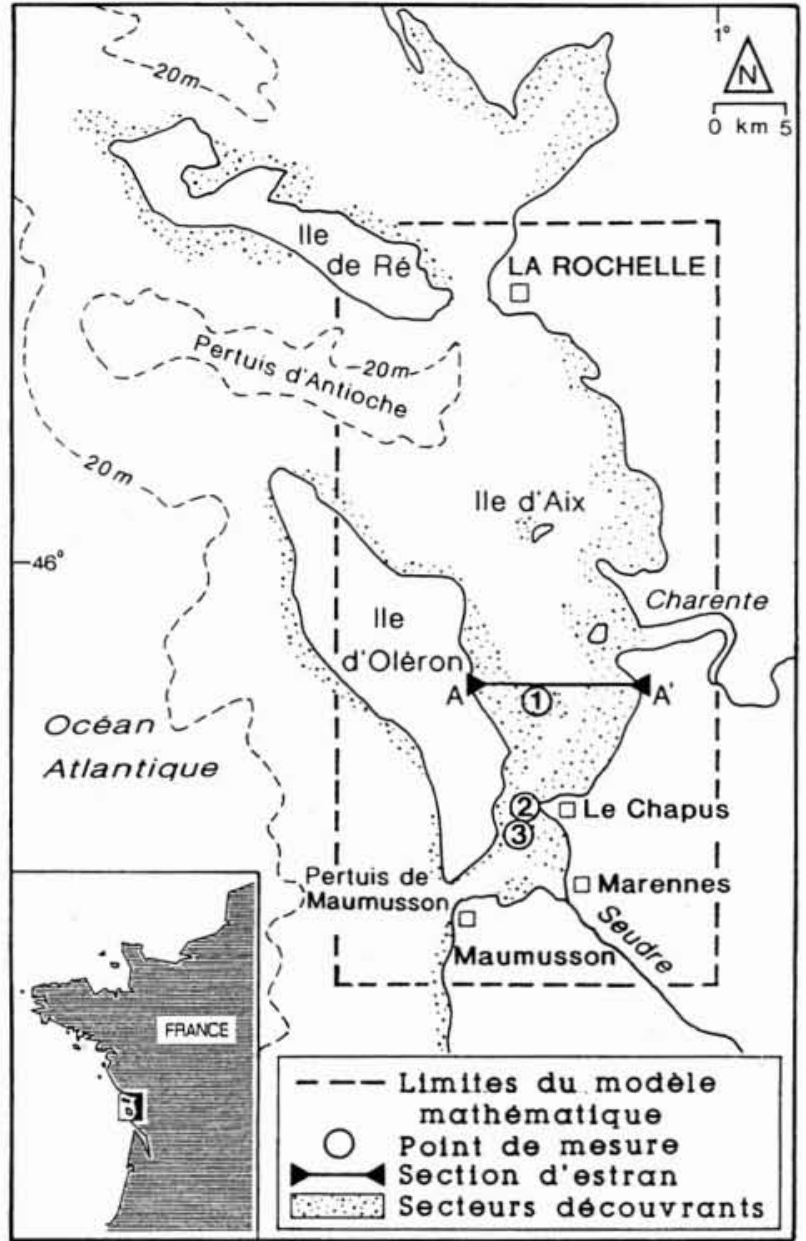

1. Localisation du bassin de Marennes-Oléron et implantation du modèle mathématique.

profondeur est de l'ordre de $20 \mathrm{~m}$ dans le pertuis d'Antioche et d'environ $5 \mathrm{~m}$ dans le reste de la baie, avec cependant de larges estrans qui bordent l'île d'Oléron et les côtes continentales. Excepté dans les secteurs de chenaux et sur le littoral nord-ouest de l'île d'Oléron, les sédiments de la baie sont de type cohésif. Entre l'axe du bassin et le continent, la fraction pélitique est comprise entre 50 et $90 \%$, tandis que vers l'île d'Oléron les sédiments deviennent plus grossiers mais la teneur en pélites reste comprise entre 10 et $30 \%$ (GoulEAU, 1975). Le régime hydrodynamique de la baie est fortement régulé par le phénomène de la marée, que le vent, les houles (ouest et nord-ouest) peuvent occasionnellement perturber. La Charente est la principale source d'eau douce du bassin, mais ses apports (jusqu'à $200 \mathrm{~m}^{3} \cdot \mathrm{s}^{-1}$ en crue) représentent $1 \%$ des débits résiduels de marée transitant par la frontière nord au niveau de l'île d'Aix. Les sédiments fins de Charente constituent un bouchon vaseux, susceptible d'être évacué en période de crues sous certaines conditions de houles et de courants. Toutefois, les sédiments fins de la baie proviennent principalement des stocks de vase océanique (GoulEAU, 1975).

\section{Présentation du modèle mathématique}

Le modèle mathématique utilisé a été initialement développé pour l'étude des transits de sédiments et des contaminants associés dans la baie de Morlaix (LE HIR et al., 1993).

\subsection{Le code de calcul}

De façon classique, le modèle de transport de sédiments cohésifs résout sous forme conservative l'équation cidessous d'advection-dispersion des MES, avec comme termes source ou puits les échanges avec le fond (éro$\operatorname{sion} E$ ou dépôt $D$ ). Le modèle est bidimensionnel horizontal $(2 \mathrm{DH})$ et calcule donc une concentration moyenne sur la verticale $(C)$.

$$
\frac{\partial h C}{\partial t}+\operatorname{div}(\vec{U} h c)=\operatorname{div}\left(K_{\mathrm{dis}} h \vec{g} \operatorname{rad} C\right)+E-D .
$$

La hauteur d'eau $h$ et le courant $U$ sont calculés à l'aide d'un modèle hydrodynamique $2 \mathrm{DH}$ qui résout les équations de Saint-Venant. La technique de résolution numérique est celle des différences finies, de type A.D.I. (SALOMON et al., 1989). Le transport des MES est calculé selon l'algorithme de Bott (in LE HIR et al., 1993).

Le frottement sur le fond, utilisé pour le calcul de $E$ et $D$ et paramétré par $u^{*}$, est pris égal au maximum entre la contribution du courant $u_{c}^{*}$ et celle de l'agitation de surface $u_{v}^{*}$. Cette formulation simpliste, qui ignore les processus d'interaction non linéaire entre la houle et les courants, est cohérente avec la non prise en compte de la direction de la houle ou du clapot, dont les propagations ne sont pas traitées dans la présente étude.

$u_{c}^{*}$ est calculé selon la loi de Strickler tandis que $u_{v}^{*}$ est calculé d'après la formulation de Jonsson (1966) :

$$
u_{r}^{*}=\sqrt{\frac{1}{2} f_{w}} u_{m}, \quad \text { où } u_{m} \text { est la vitesse orbitale. }
$$

Selon un modèle de houle de Stokes au $1^{\text {er }}$ ordre (qu'on admettra valide pour les clapots), compte tenu des faibles hauteurs d'eau devant les longueurs d'ondes, et en choisissant une valeur moyenne de 0,07 pour $f_{w}$, on obtient finalement : $u_{v}^{*} \approx 0,3 \mathrm{H} / \sqrt{h}$.

En fonction des hypothèses précédentes, le forçage houle ou clapot intervient seulement par la hauteur locale des vagues et la profondeur. Dans le cadre des tests de faisabilité que nous présentons dans cet article, cette hauteur sera imposée.

\subsection{Comportement des sédiments}

La loi de dépôt des MES est celle proposée par KRONE (1962) :

$$
D\left(\mathrm{~kg} \cdot \mathrm{m}^{-2} \cdot \mathrm{s}^{-1}\right)=W_{s} \cdot C_{f} \cdot\left(1-\left(u^{*} / u_{d}^{*}\right)^{2}\right)
$$

où : $u_{d}^{*}$ est la vitesse de frottement au-delà de laquelle le dépôt est nul ; $W_{s}$, la vitesse de chute, peut varier en fonction de la concentration en MES et de la turbulence (paramétrée par $u^{*}$ ), afin de tenir compte de possibles phénomènes de floculation (LE HIR et al., 1993); 
$C_{f}$, la concentration près du fond, est estimée à partir de la concentration moyenne en faisant l'hypothèse d'un profil d'équilibre de type Rouse.

Pour l'érosion, c'est la formulation classique des sols consolidés qui est retenue, pour la simplicité de son paramétrage :

$$
E\left(\mathrm{~kg} \cdot \mathrm{m}^{-2} \cdot \mathrm{s}^{-1}\right)=E_{0}\left(\left(u^{*} / u_{e}^{*}\right)^{2}-1\right) .
$$

Cependant la formulation fait apparaître $u_{c}^{*}$, la vitesse de frottement critique à partir de laquelle le sédiment commence à être érodé. Migniot (1989) a proposé une relation entre $u_{c}^{*}$ et la rigidité initiale $\tau_{y}$ du sédiment, paramètre rhéologique lui-même fonction de la densité.

C'est un modèle de tassement, discrétisant le sol en couches fines au droit de chaque maille du modèle de transport sédimentaire 2DH, qui permet de suivre l'évolution du profil vertical de densité et simultanément de gérer le stock sédimentaire en intégrant des flux d'érosion et de dépôt. Ce modèle, décrit dans LE HIR et al. (1989), rend compte des processus de dessèchement et de gonflement successifs des vases lors des cycles d'émersion/immersion qui peuvent entraîner des modifications notables de l'érodabilité du sédiment au cours d'un cycle de marée.

\subsection{Implantation du modèle sur le secteur de Marennes- Oléron}

L’extension géographique du modèle (fig. I) a été choisie de telle sorte que l'incertitude sur les conditions aux limites, en particulier le flux de MES rentrant, ne pèse pas trop sur les résultats dans le bassin. Le choix d'une maille de $1000 \mathrm{~m}$ de côté résulte d'un compromis entre la nécessaire finesse de description de la bathymétrie, en particulier pour les secteurs de chenaux, et la recherche d'un coût d'exploitation réduit, en vue de simulations de longue durée pour refléter les variations saisonnières des bilans sédimentaires.

Quant au modèle de tassement, il peut gérer l'évolution de 200 couches ayant une masse de sédiment sec fixée à $0,1 \mathrm{~kg} \cdot \mathrm{m}^{-2}$. correspondant à des épaisseurs de l'ordre du $\mathrm{mm}$ en fonction de la densité du sédiment.

Les conditions hydrodynamiques aux limites ouvertes sont des niveaux de marée, exprimés par trois constantes harmoniques, issus d'un calcul à l'échelle du golfe de Gascogne. Une modélisation sinusoïdale des constantes harmoniques rend compte schématiquement du cycle vive eau/morte eau. Par ailleurs le débit de Charente est pris constant, égal à $25 \mathrm{~m}^{3} \cdot \mathrm{s}^{-1}$. Pour les MES, les conditions aux limites sont

- un flux nul en amont de la Charente,

- au large en période de flot, une concentration uniforme et constante égale à $10 \mathrm{mg} \cdot \mathrm{I}^{-1}$, conformément aux observations in situ (BACHER, 1989).

En ce qui concerne les conditions initiales, la principale difficulté est la définition du fond vaseux (quantité et état de consolidation). S'inspirant de la cartographie sédimentaire de SAuriau et al. (1989), des stocks de vase infinis sont introduits dans les mailles des secteurs de vase pure et de vase sableuse, avec une densité initiale uniforme assez élevée. Ailleurs, en particulier dans tous les secteurs de chenaux, les stocks initiaux de vases sont choisis nuls.
Le paramétrage hydraulique et sédimentaire est résumé ci-dessous :

$k$ : coefficient de frottement (de type Strickler) $=33$

$u_{d}^{*}:-0,04 \mathrm{~m} \cdot \mathrm{s}^{-1}$

$w_{s}$ : varie de 0,5 à $5 \mathrm{~mm} . \mathrm{s}^{-1}$ en fonction de l'état de floculation

$E_{0}: 7 \cdot 10^{-5} \mathrm{~kg} \cdot \mathrm{m}^{-2} \cdot \mathrm{s}^{-1}$

$\tau_{y}=1,5 \cdot 10^{-9} \cdot C_{\text {sed }}^{3.45}$, relation calibrée en fonction de données expérimentales de rigidité initiale obtenues sur des prélèvements dans le bassin (LHF, 1992).

La loi de consolidation des vases n'a pas été calibrée sur des données expérimentales, mais conduit à des courbes de tassement similaires aux courbes expérimentales obtenues dans des colonnes à sédimentation (LHF. 1992).

\section{Résultats du modèle}

Les simulations ont été effectuées sur des périodes de l'ordre d'un mois, soit plusieurs cycles vive eau/morte eau, les premiers étant destinés à réduire l'effet des conditions initiales : ainsi les résultats présentés sont-ils quasi-périodiques.

\subsection{Calculs hydrodynamiques}

La simulation des effets de marée est validée par une dizaine de mesures de courant réalisées en 1971 (RAllLARD et al., 1993). A titre d'exemple, la figure 2 montre un accord relativement correct entre mesures et modèle. les différences pouvant être imputées à un manque de représentativité des mesures par rapport à la dimension des mailles, autant qu à une éventuelle imprécision du modèle.

Les champs de courant présentés en figure 3 illustrent la complexité des cicrulations dans les Pertuis, en particulier la convergence des courants de flot, l'avance de phase des secteurs de faible profondeur, l'effet du découvrement etc. L'excursion des masses d'eau au cours d'une marée de vive eau, de l'ordre de $5 \mathrm{~km}$ entraîne un assez fort mélange par dispersion des éléments dissous, tandis qu'une circulation résiduelle intense vers le sud provoque un renouvellement rapide des masses d'eau par le pertuis d'Antioche, avec un temps de séjour de l'ordre de 3 à 6 jours dans le bassin ostréicole (BACHER, 1989).

\subsection{Matières en suspension}

Les mesures de MES dans le bassin sont assez rares et trop discontinues pour être véritablement exploitables, sauf au Chapus (station 2, fig. I) où des campagnes de mesure en continu de la turbidité (et d'autres paramètres biochimiques) ont été réalisées en 1988, 1990 et 1991. Ce point central a aussi l'intérêt d'être représentatif de processus se produisant dans les deux moitiés Nord et Sud du bassin de Marennes, et finalement du niveau de turbidité global du secteur. C'est donc en ce point que nous présentons les résultats du modèle (fig. 4). 

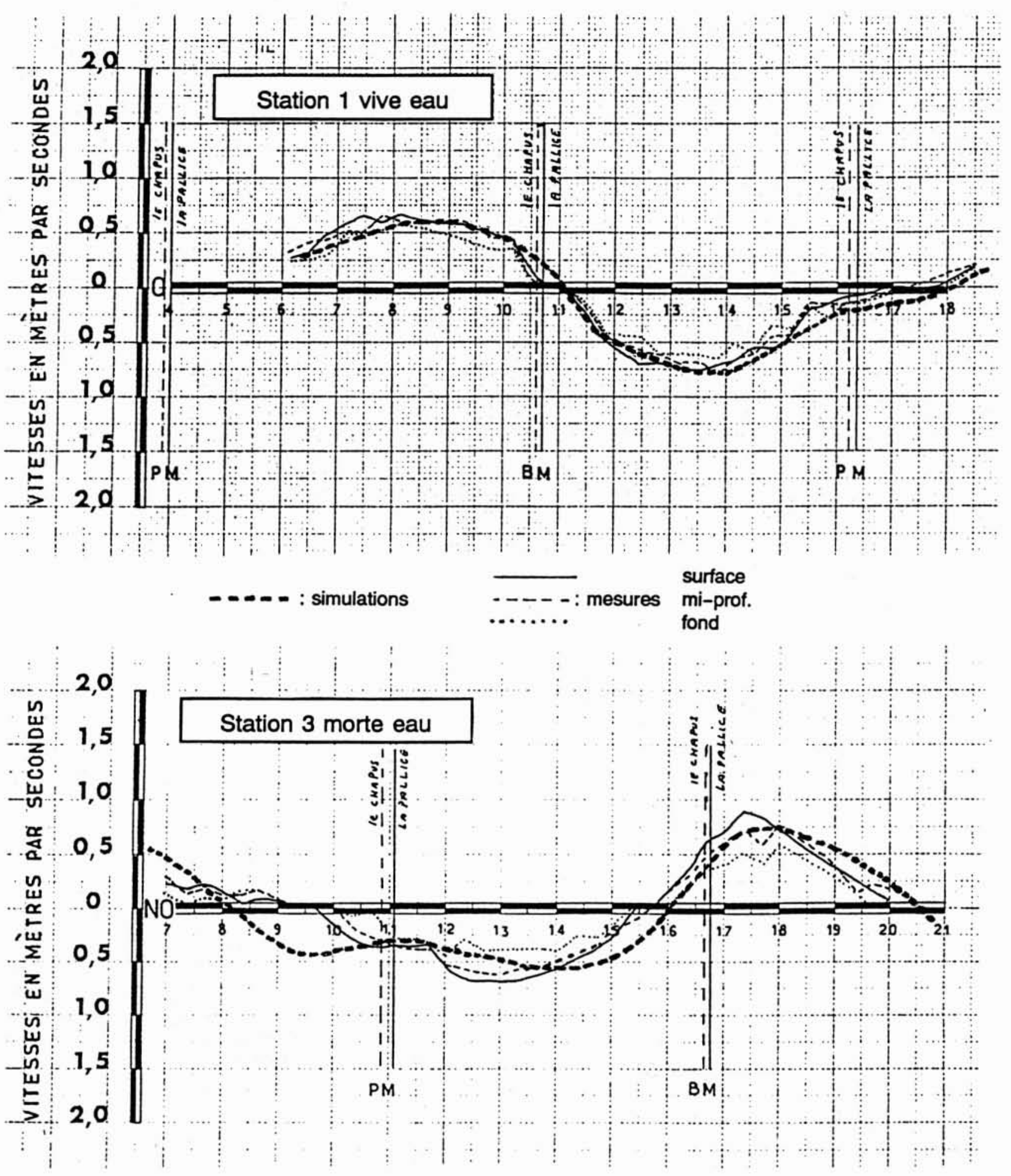

2. Comparaison entre courants mesurés et courants calculés.

On observe que les niveaux de turbidité, mesurés en 1990 dans des conditions météorologiques calmes, sont bien reproduits, tant en vive eau (environ $200 \mathrm{mg} \cdot \mathrm{l}^{-1}$ ) qu'en morte eau (de l'ordre de $70 \mathrm{mg} .1^{-1}$ ).

A l'échelle de variation de la marée, l'évolution mesurée des MES passe par deux maxima. Le premier dont l'amplitude est relativement indépendante du coefficient de la marée, se produit au début du flot et correspond au passage des suspensions en provenance du sud du bassin. Le second maximum se produit après la renverse des courants et résulte des remises en suspension des vases des estrans situés au nord. La valeur de ce pic 

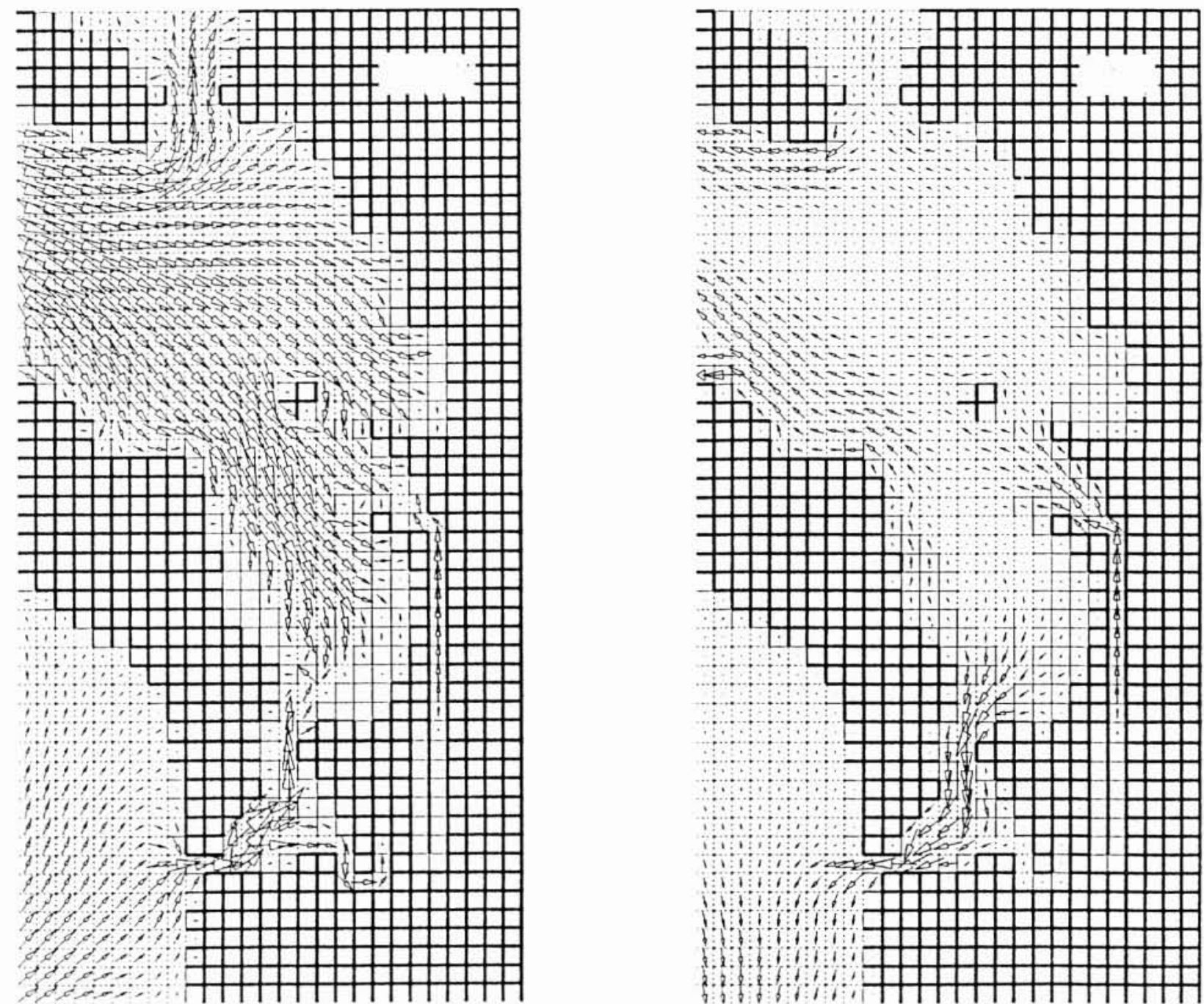

3. Exemples de champs de courants de vive eau calculés par modéle mathématique

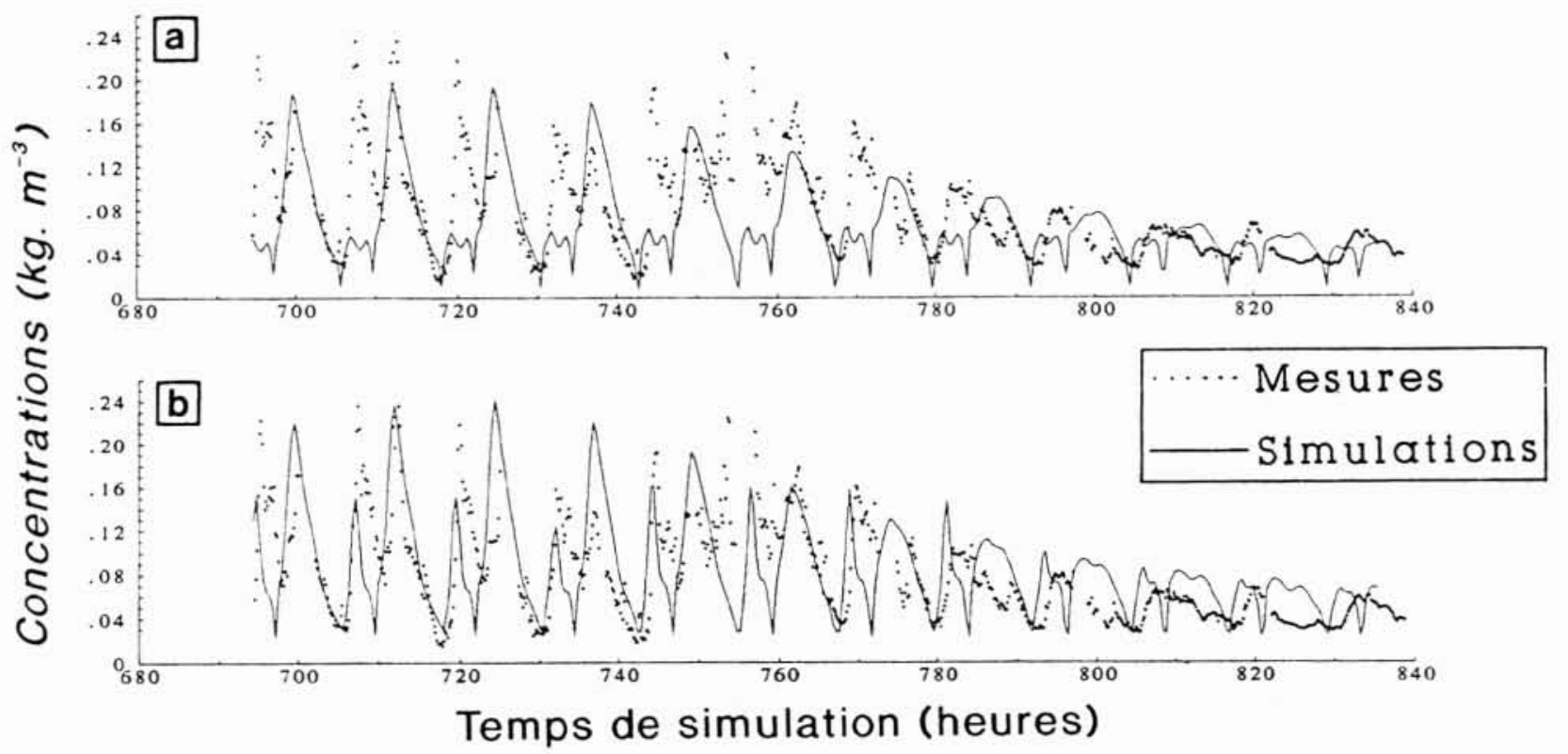

4. Evolution des matières en suspension pendam o jours à la station du Chapus (campagne 1990). Comparaison entre mesures et modèle. a) Simulation en condition de marée seule, b) Simulation en condition de marée + clapot. 
est étroitement liée au coefficient de marée. Le modèle révèle également la présence de deux pics de turbidité pendant le cycle de marée, mais l'écart avec les mesures demeure significatif surtout durant les marées de viveeau.

Le poids important du premier pic pendant les observations en marée moyenne incite à penser à une éventuelle action d'un clapot susceptible de mettre en mouvement les sédiments déposés dans les secteurs très peu profonds qui constituent les estrans, alors que les courants y sont faibles et que la houle ne s'y propage plus (en raison des réfractions/diffractions et déferlements successifs). De tels clapots générés par un vent relativement faible sont observés fréquemment.

Nous avons donc considéré une simulation cumulant les effets de la marée et ceux d'un clapot de $5 \mathrm{~cm}$ de hauteur sur l'ensemble du bassin. Lorsque la profondeur est inférieure à $5 \mathrm{~cm}$, la hauteur du clapot supposé " déferler » est définie égale à la profondeur. Les résultats au Chapus (fig. 4b) sont alors nettement améliorés, avec des pics de début de flot bien marqués.

Il est également possible que la supériorité de ces maxima de basse mer sur ceux qui sont corrélés au courant nord-sud, supériorité non reproduite par le modèle, soit due à la proximité entre le point de mesure et l'estran est, que naturellement le maillage grossier du modèle ne permet pas de reproduire.

\subsection{Bilans sédimentaires}

Nous abordons ce thème sous deux formes synthétiques : l'évolution temporelle des stocks de sédiments, dans l'eau et sur le fond, intégrés sur l'ensemble du secteur d'étude (fig. 5) et une cartographie des variations sédimentaires au cours d'un cycle vive eau/morte eau (fig. 6). Deux calculs ont été considérés : la simulation d'un cycle de marée sans effet d'agitation et celle du même cycle de marée avec clapot permanent et uniforme de $5 \mathrm{~cm}$ de hauteur.

Les évolutions de stock ne sont présentées que pour le deuxième cas, marée + clapot, simulation jugée meilleure selon les résultats au Chapus. La figure 5 met en évidence la modulation par l'amplitude de marée des échanges entre sédiment et MES, une forte fluctuation de ces dernières pendant les marées de vive eau, presqu'autant qu'entre vive eau et morte eau. Ceci est révélateur d'un dépôt quasi-total et uniforme des matériaux aux étales de vive eau. On note aussi un retard des quantités de MES sur l'amplitude de la marée, que semble confirmer la mesure au Chapus (fig. 4). Enfin on remarque une légère

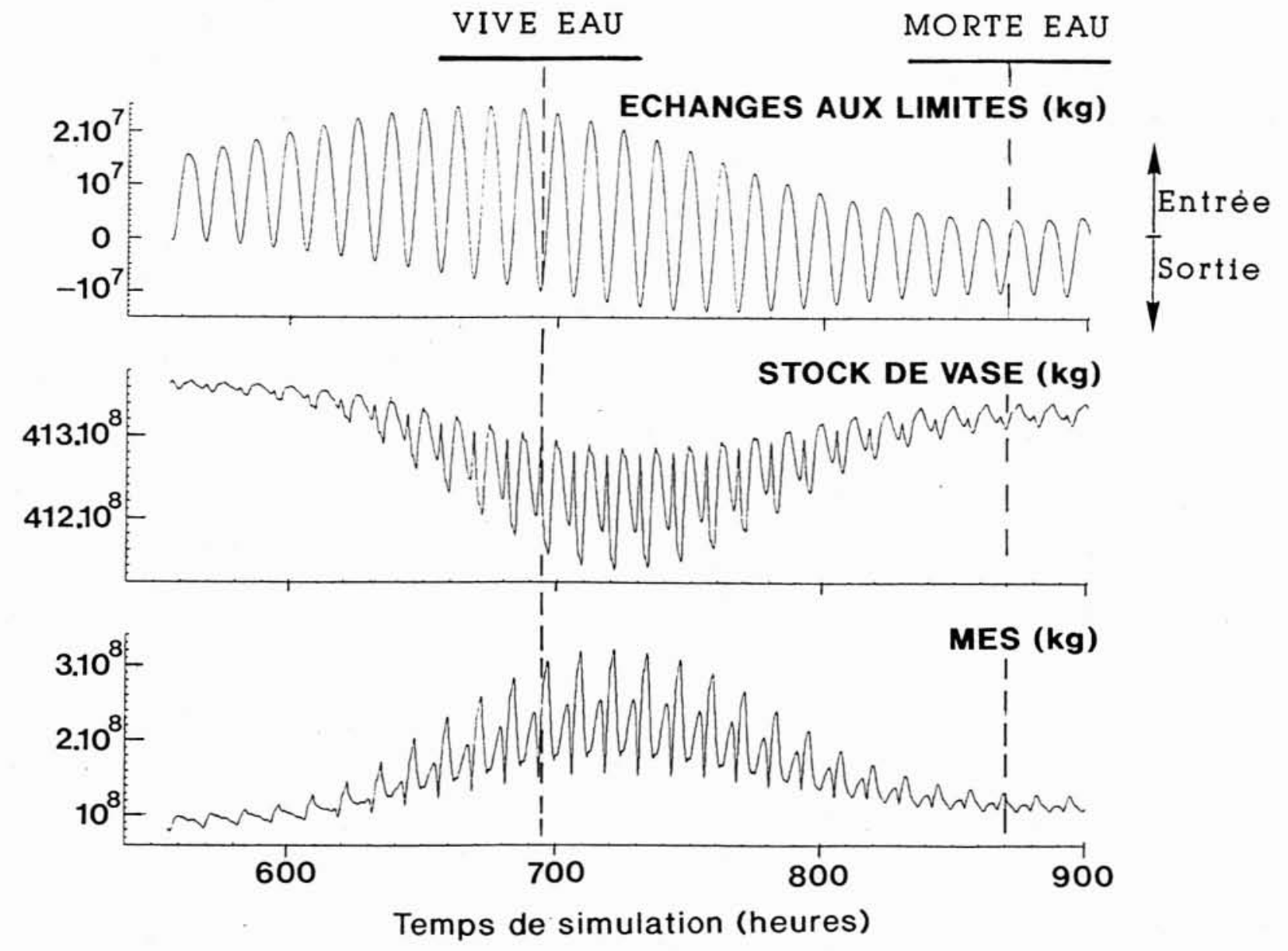

5. Bilan sédimentaire : évolution des stocks pendant un cycle de marée (action de la marée et d'un clapot uniforme de $5 \mathrm{~cm}$ de hauteur). N.B. : La valeur du stock de vase est dépendante des conditions initiales : seule la variation pendant le temps de simulation est à prendre en considération. 




6. Bilan sédimentaire : variation des stocks de vase après un crele semi-lumairé

a) Action de la marée seule.

b) Action de la maréc et d'un clapot uniforme de $5 \mathrm{~cm}$ de hauteur.

dérive entre les deux mortes eaux successives, révélatrice d'un accroissement des MES qui n'était pas perceptible en l'absence de clapot. De la même façon les échanges aux frontières montrent une fuite des sédiments du bassin, mais les quantités en jeu sont faibles. D'autre part, il faut rappeler le caractère arbitraire du clapot uniforme et permanent qui pourrait (devrait?) être modulé : le modèle montre en fait une forte sensibilité à ce forçage, tout au moins en terme de bilan.

L'évolution des fonds sédimentaires sous l'action seule de la marée, après 14 jours (fig. $6 a$ ) montre peu de mouvements sédimentaires dans le pertuis d'Antioche et un déplacement de la vase du bas de l'estran vers le haut dans le bassin de Marennes. Par ailleurs, le secteur océanique à l'ouest du pertuis de Maumusson est le siège d'un dépôt faible mais étendu. L'action du clapot modifie peu ce schéma (fig. 6b) sauf sur le haut des estrans où les dépôts sont moindres. Corrélativement, de la vase apparaît dans le chenal au sud du bassin en provenance des enlèvements sur estran.

Cette évolution contrastée de l'estran est mise en évidence sur la figure 7 qui représente les profils successifs de variation cumulée des stocks de vase, le long de la section $A A^{\prime}$ du bassin. On note une évolution très sensible de l'estran est (vasière de Brouage) avec en vive eau une forte érosion au bas de l'estran et un dépôt important à l'est, puis une érosion liée au clapot au niveau de la maille la plus haute (extrémité est). 


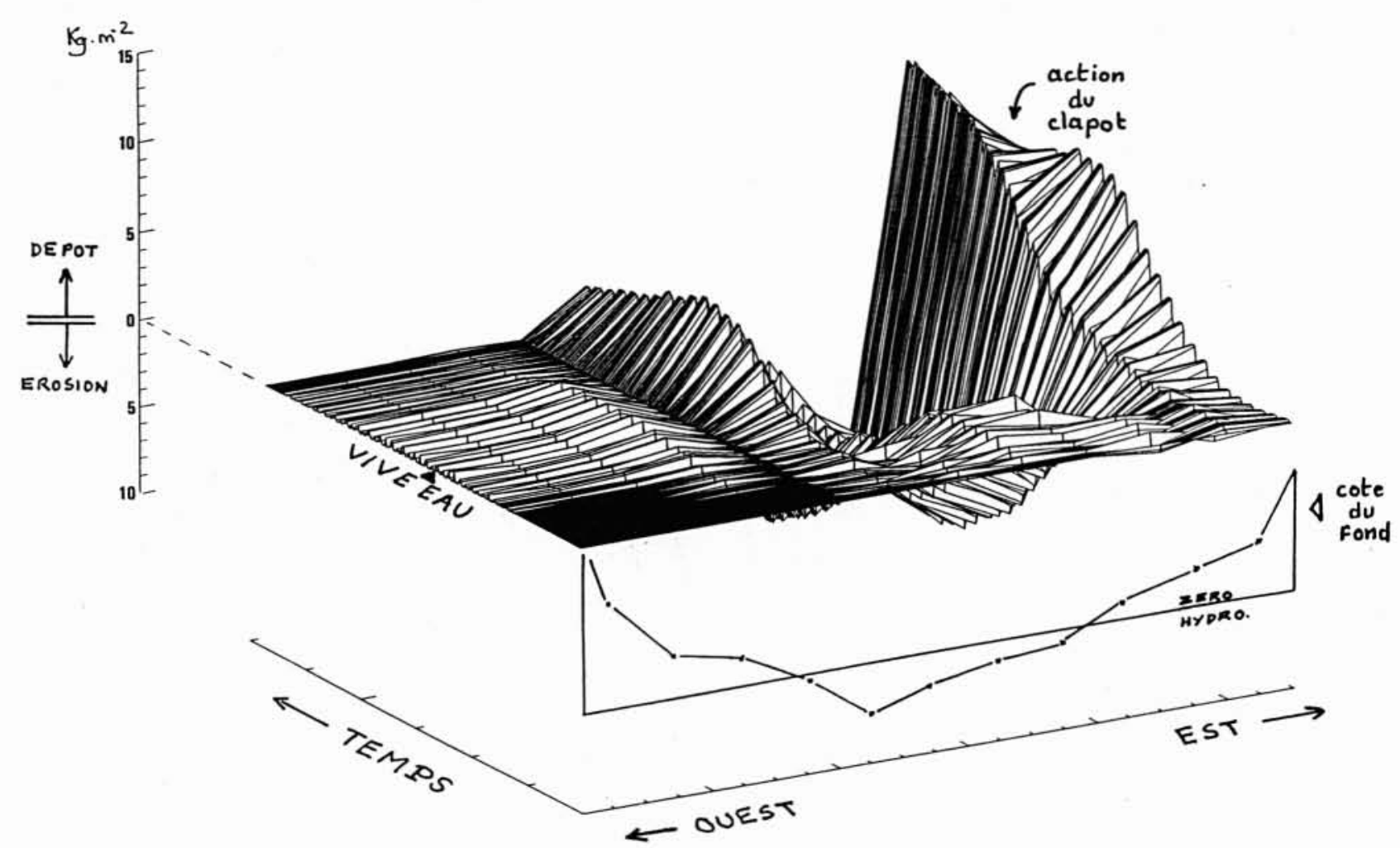

7. Evolution des dépôts/érosions le long d' une section transversale du bassin de Marennes-Oléron, entre le Chapus et la Charente (fig. I. section $\left.A A^{\prime}\right)$, simulée pendant un cycle de marée en présence de clapot.

Les observations de Gouleau (1975) confirment le déplacement des sédiments fins vers le haut de l'estran, où l'engraissement serait de $3,5 \mathrm{~cm} / \mathrm{an}$. De même, la comparaison des bathymétries de 1970 à 1985 effectuée un peu plus au sud par la DDE de Charente Maritime indique une érosion de l'ordre du mètre en bas d'estran.

Le modèle semble donc reproduire qualitativement les processus observés, mais en les surestimant puisque les quantités de vases en jeu correspondent à des épaisseurs moyennes de $1,5 \mathrm{~cm}$ (dépôt ou érosion) en 15 jours.

\section{Discussion.}

Si le modèle semble reproduire correctement le régime des MES, il génère des mouvements de vase a priori excessifs, malgré le manque de données de validation à échelle de temps comparable à celle du modèle. En particulier certains secteurs réputés vaseux, comme celui situé à $3 \mathrm{~km}$ au sud de l'Ile d'Aix « seraient » toujours en érosion. La simulation d'une houle schématique, non représentée ici faute de place, a contribué à compenser cet effet de marée mais partiellement seulement, montrant toutefois la nécessité de poursuivre les investigations sur les effets de la houle.

Egalement il serait nécessaire de mieux prendre en compte les processus sédimentaires dans l'estuaire de la Charente, tout proche de ce secteur sensible.
On peut aussi remarquer le manque de transition entre les zones en érosion et celles en dépôt (p. ex. figure 7), probablement lié à la discrétisation trop grossière du modèle. Un raffinement des mailles améliorerait certainement les résultats, et permettrait en outre une comparaison plus pertinente de ces résultats avec les mesures ponctuelles. Un autre point sensible est la définition des conditions initiales, qui est d'autant plus délicate que les caractéristiques rhéologiques des vases du bassin sont connues pour être hétérogènes. Une " couverture rhéologique " (résistance au cisaillement, densité superficielle) plusieurs fois renouvelée serait sans doute utile.

Plus généralement une étude de sensibilité du modèle au paramétrage (lois de dépôt, de tassement et d'érosion), en cours, doit confirmer la robustesse des principaux résultats du modèle vis-à-vis de ces multiples paramètres.

\section{Conclusion}

Le premier objectif de l'étude, qui consiste à décrire la variabilité spatio-temporelle des turbidités dans le bassin en vue d'en examiner l'impact sur la production biologique (primaire et conchylicole) semble atteint.

Les rôles respectifs de la marée et des agitations de surface ont pu être montrés, à l'aide de cas tests pour les dernières. Des pistes pour la progression de l'étude sont ainsi dégagées, à savoir la nécessité de traiter la propaga- 
tion des houles dans le bassin, la génération du clapot, l'effet du déferlement sur les estrans, la modulation des courants sous l'effet du vent et du déferlement des houles. Enfin et surtout, ce travail que l'on peut qualifier de "préliminaire" a mis en évidence les lacunes en ce qui concerne les mesures de calibration et de validation nécessaires, en particulier sur l'évolution de l'estran sous l'effet d'un forçage combiné houle/clapot/courant.

\section{Références}

BACHER C.. 1989. - Capacité trophique du bassin de Marennes-Oléron : couplage d'un modèle de transport particulaire et d'un modèle de croissance de l'huitre C. gigas. Aqua. Living ressources, 2, 199-214.

GOUleAu D., 1975. - Les premiers stades de la sédimentation sur les vasières littorales atlantiques. Rôle de l'émersion. Thèse de $3^{e}$ cycle. Université de Nantes, 241 p.

héral. M., Deslous-Paoli J.M., Razet D. et Prou J., 1984. - Essai de mise en évidence in situ de paramètres biotiques et abiotiques de l'eau et de l'interface eausédiment intervenant dans la production de l'huitre Crassostrea gigas. Océanis, 10 (4) : 465-475.

Le Hir P., Bassoullet el L'Yavanc J., 1989. - New developments about mud transport models : application to a macrotidal estuary. Proc. Int. Symp. on sediment transport modeling. New Orleans, Aug. 14-18, 1989. Ed. S.S.Y. Wang, Pub. ASCE.

Le Hir P., Bassoullet P. et L'Yavanc J., 1993. - Application of a mutivariable transport model for understanding, cohesive sediment dynamics. In nearshore and estuarine cohesive sediment transport. A.J. Mehta (Ed.), Coastal and Estuarine Studies, Vol. 42, A.G.U. (Pub.).

LHF, 1992. - Caractéristiques mécanique des vases et des biodépôts de Marennes-Oléron. Rapport d'étude. Laboratoire d'Hydraulique de France, $14 \mathrm{p}$.

Migniot C., 1989. - Tassement et rhéologie des vases. La Houille Blanche, 2: 95-111.

RaILLARD O.. 1991. - Etude des interactions entre les processus physiques et biologiques intervenant dans la production de l huître Crassostrea gigas du bassin de MarennesOléron. Thèse doctorat Univ. Paris, 203 p.

RAIlLARD O., LE HIR P., 1993. - Hydrodynamical, sedimentation and erosion model of the hay of Marennes-Oléron : presentation of the main results. Rapport contrat CEE, IFREMER (en français). $\mathrm{N}^{\circ} \mathrm{AQ} 2500, \mathrm{n}^{\circ} 4$, mai 93.

Salomon J.C., Pommepuy M., Breton M., Derrien A., 1989. - Modèle mathématique de la dispersion des bactéries entériques en baie de Morlaix. Symposium "Modèles mathématiques pour la gestion de la qualité des eaux " organisé par la Société Hydrotechnique de France, novembre 1989.

Sauriau P.G., Mouret V., Rince J.P., 1989. Organisation trophique de la malacofaune benthique non cultivée du bassin ostréicole de Marennes-Oléron. Oceanologica Acta, 12, 2: 193-204. 